\title{
Caroline Émilie « Lili » Bleeker
}

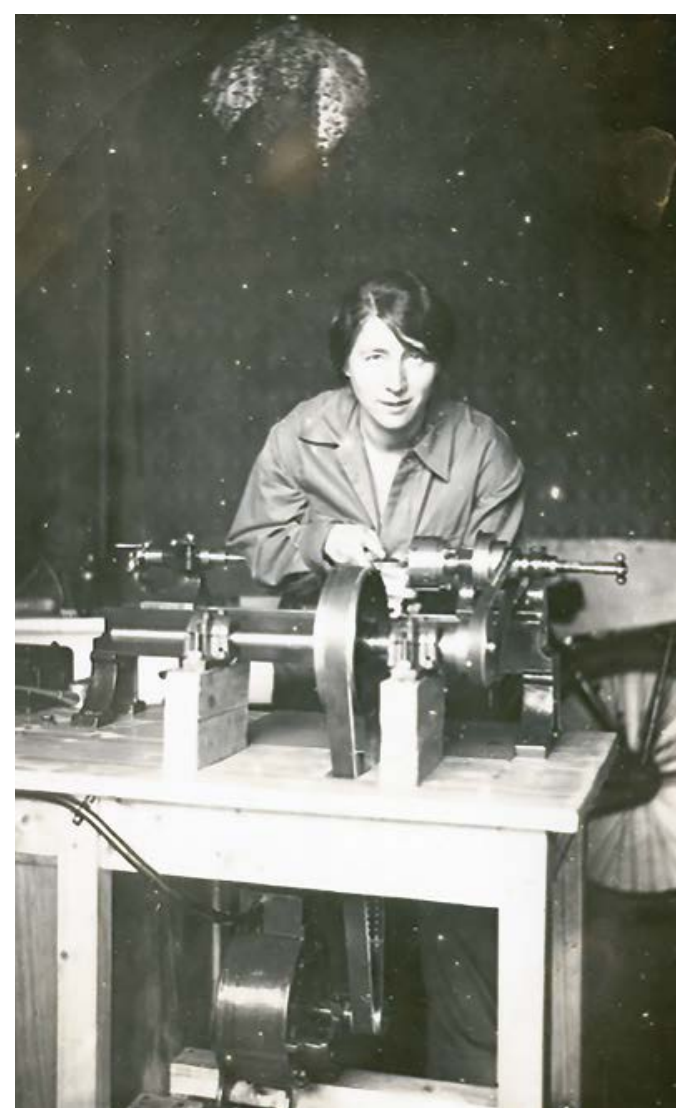

LUCie LEBOULLEUX

LESIA, Observatoire de Paris, Université PSL, CNRS, Université de Paris, Sorbonne Université, 5 place Jules Janssen, 92195 Meudon, France

* lucie.leboulleux@obspm.fr

Caroline Émilie « Lili » Bleeker était une physicienne et auto-entrepreneuse néerlandaise. Après un doctorat sur les spectres des alcaloïdes, elle monte la première entreprise néerlandaise de production de matériel et d'instruments optiques. Elle signe notamment avec Frederik Zernike le brevet sur le microscope à contraste de phase, pour lequel le physicien obtient le prix Nobel de Physique en 1953. Elle se démarque également pour son engagement contre le nazisme lors de la Seconde Guerre Mondiale.

\section{https://doi.org/10.1051/photon/202010322}

Article publié en accès libre sous les conditions définies par la licence Creative Commons Attribution License CC-BY (http://creativecommons.org/licenses/by/4.0), qui autorise sans restrictions l'utilisation, la diffusion, et la reproduction sur quelque support que ce soit, sous réserve de citation correcte de la publication originale.

\section{Enfance et éducation}

Caroline Émilie «Lili» Bleeker naît le 17 janvier 1897 dans la ville de Middelburg au Pays-Bas. Elle est alors la cinquième et dernière enfant de Johannes Lambertus Bleeker, un pasteur évangélique luthérien, et de Gerhardina Martha Döhne. Si ses sœurs quittent le foyer pour devenir enseignantes, Caroline
Bleeker, en tant que benjamine, est supposée rester auprès de ses parents afin d'aider sa mère au quotidien. Cependant, elle s'oppose rapidement à ce destin et se lance dans des études supérieures.

En 1916, après son diplôme de Hogere Burgerschool (baccalauréat), Caroline Bleeker commence à étudier les mathématiques avant de se

BIOGRAPHIE

\begin{tabular}{lll|l}
\hline I & $\mid$ & $\mid$ & $\mid$ \\
17 Janvier 1897 & 1930 & 1939 & 1960 \\
$\begin{array}{l}\text { Naissance à } \\
\text { Middelburg, }\end{array}$ & Création de sa & Société NEDOPTIFA & Dépôt du brevet sur le \\
Pays-Bas & société de conseils & & Décès à Zeist, \\
microscope à contraste \\
de phase
\end{tabular}


tourner vers la physique à l'université d'Utrecht. Elle se spécialise ensuite en astronomie puis en physique expérimentale et théorique. A la suite de son master et grâce à un travail d'assistante au laboratoire de Physique de l'université d'Utrecht, elle se lance alors dans un doctorat sous la direction du professeur et docteur Leonard S. Ornstein. Le 5 novembre 1928, elle devient docteure avec distinctions en physique expérimentale. Sa thèse, intitulée Emissie- en dispersiemetingen in de seriespectra der alkalieën (mesures d'émission et de dispersion dans les spectres en série des alcaloïdes), est imprimée par G. J. Willemse, dont le fils Gerard deviendra son principal associé et compagnon de vie.

\section{Caroline Bleeker, l'auto-entrepreneuse}

Malgré les recommandations élogieuses de Leonard Ornstein, Caroline Bleeker peine à trouver un emploi après sa thèse. Finalement, un an et demi après son doctorat, elle fonde un cabinet de consultation en physique qui produit du matériel de laboratoire et des instruments de mesure. Si le cabinet est originellement localisé dans son propre salon, il s'étend rapidement et finit par déménager dans une petite usine à Utrecht. En 1935, Gerard Willemse rejoint la société dont il devient co-directeur.

Un an plus tard, grâce à plusieurs investisseurs dont le professeur Frederik « Frits » Zernike (voir Photoniques 73 - 2014), Caroline Bleeker monte aussi un atelier d'optique et l'entreprise commence à produire et distribuer du matériel et des instruments optiques. Notamment, Caroline Bleeker négocie et obtient le marché des jumelles à prismes pour l'armée néerlandaise, et son produit est même présenté au premier ministre néerlandais Hendrikus « Hendrik » Colijn.

En 1939, le cabinet de conseil compte plusieurs dizaines d'employés et évolue pour devenir la Nederlandsche Optiek- en Instrumentenfabriek Dr. C.E. Bleeker, aussi appelée NEDOPTIFA ou BLEEKER. En sus de l'armée, on trouve parmi ses principaux clients des scientifiques, des professeurs d'universités et de grands laboratoires.

\section{Seconde Guerre Mondiale}

Le 10 mai 1940, afin de contourner la ligne Maginot, l'armée allemande lance une attaque aussi soudaine qu'inattendue contre les Pays-Bas. C'est la célèbre Bataille de Hollande, qui ne dure alors qu'une semaine et aboutit à la capitulation du pays. Mais Caroline Bleeker choisit son camp : afin d'éviter que ses produits soient utilisés par l'armée allemande, elle stoppe la production des jumelles à prismes et, en contrepartie, développe celle de microscopes dédiés aux universités. L'entreprise connaît alors des difficultés : les ventes chutent drastiquement et Caroline Bleeker doit utiliser ses propres revenus pour payer ses employés. Par ailleurs, elle cache des citoyens de confession juive dans son usine d'Utrecht mais, dénoncée aux autorités en 1944, elle les aide à s'enfuir avant de disparaître à son tour avec son compagnon. Jusqu'à la fin de la guerre, l'entreprise restera fermée et l'usine sera occupée par les troupes allemandes et néerlandaises.

\section{L’après-guerre}

Au sortir de la guerre, en mai 1945, la société est au plus mal et ne peut recevoir d'aide de l'état pour se redresser totalement. Malgré ces difficultés, Caroline Bleeker et Gerard Willemse ouvrent une seconde usine dans la ville de Zeist, qui est inaugurée par le ministre de la reconstruction et du logement, Joris in't Veld. Caroline Bleeker et Frits Zernike déposent aussi un brevet sur le microscope à contraste de phase, un instrument optique non invasif permettant d'étudier la structure des cellules, que l'entreprise NEDOPTIFA entreprend de produire. C'est d'ailleurs pour cette invention que Frits Zernike reçoit le prix Nobel de Physique en 1953. Enfin, en 1961, la reine Juliana des Pays-Bas visite l'usine de Zeist et se voit offrir des jumelles produites par la société et gravées aux armoiries royales. Malgré tout, les dettes accumulées au cours de la guerre et accrues par l'achat de la nouvelle usine ne se comblent pas.

\section{Dernières années}

Le 31 décembre 1963, à respectivement 66 et 63 ans, Caroline Bleeker et Gerard Willemse se retirent de la direction de leur entreprise et une nouvelle direction est nommée. La société fusionne avec «De Oude Delft » en 1969 avant de fermer définitivement en 1978.

Les dernières années de sa vie, Caroline Bleeker souffre d'une forme grave d'Alzheimer qui nécessite beaucoup de soins. Le 8 novembre 1985, elle décède à Zeist où elle est enterrée aux côtés de son 
compagnon, parti cinq ans plus tôt d'une maladie cardio-vasculaire. Cependant, le nom de la physicienne n'est inscrit sur leur tombe commune qu'en 1997. Cette tentative de faire disparaître Caroline Bleeker de l'Histoire est réitérée lors de son oraison funèbre, au cours de laquelle ses exploits et son entreprise ne sont pas mentionnés.

\section{Le microscope à contraste de phase}

Parmi les travaux marquants réalisés par Caroline Bleeker, on compte la conception du microscope à contraste de phase dont elle a co-signé le brevet avec Frederik Zernike (Inrichting voor microscopische waarneming met variabel fasecontrast, en microscoopobjectief, geschikt voor deze inrichting, brevet $\mathrm{n}^{\circ}$ 94480, 1960).

Dédié à la bactériologie et à l'étude des cellules et micro-organismes transparents, ce microscope permet de s'affranchir des méthodes traditionnelles invasives, qui utilisent des colorants fixateurs. Il utilise les différences d'indices optiques des objets transparents à observer, qui engendrent des déphasages

\section{On peut se souvenir de} Caroline Bleeker comme d'une experte en physique expérimentale et d'une entrepreneuse hors-pair, deux milieux qui étaient, à l'époque, presque exclusivement masculins.

\section{Récompenses}

Caroline Bleeker ne s'est pas illustrée qu'en physique : pour son engagement contre le nazisme et sa bravoure, elle reçoit en 1946 une distinction royale en devenant officier de l'Ordre d'Orange-Nassau (Officier

, pour sa contribution à la physique et l'optique, le bâtiment du groupe de Physique Instrumentale de l'Université d'Utrecht porte son nom depuis 1998 : Caroline Bleekergebouw.

\section{Aujourd'hui}

On peut se souvenir de Caroline Bleeker comme experte en physique expérimentale et d'une entrepreneuse hors-pair, deux milieux qui étaient, à l'époque, presque exclusivement masculins. Son refus des conventions et des restrictions dues à son genre en font un modèle féminin original et toujours d'actualité : dans sa vie professionnelle, elle était consciente de ses capacités intellectuelles et se refusait à accepter une position inférieure à ses connaissances et dans la sphère privée, elle rejetait les conventions sociales établies telles que le mariage ou l'atta(ou retards) entre les ondes lumineuses les traversant. L'œil et les détecteurs n'étant pas directement sensibles à ces différences de marche, le microscope à contraste de phase les transforment en variations d'intensité. Pour cela, il faut que le rayon à détecter interfère avec un rayon de référence. Frederik Zernike propose d'imposer un déphasage de $\pi / 2$ (ou un quart de longueur d'onde) au rayon de référence en utilisant des anneaux ou une lame de phase. Caroline Bleeker et Frederik Zernike conçoivent le premier modèle de microscope à contraste de phase et NEDOPTIFA est la première entreprise à le commercialiser. chement de la benjamine au foyer parental.

Loin de rester symbolique, son combat pour les droits des femmes s'est traduit de façon concrète : elle était attachée à l'embauche de femmes parmi ses employés et une section du journal de son entreprise s'adressait spécifiquement aux femmes : «Van vrouw tot vrouw » (de femme à femme).

Ainsi, par sa personnalité, ses accomplissements et ses engagements, Caroline Bleeker est devenue une figure non seulement de l'optique, mais aussi de l'émancipation des femmes et de leur mise en lumière tardive dans un milieu traditionnellement masculin. 